\title{
Experimentation and direct numerical simulation of self-similar convergent detonation wave
}

\author{
R. Sorin ${ }^{\text {a }}$, C. Matignon, and O. Bozier
}

CEA, DAM, DIF, 91267 Arpajon, France

\begin{abstract}
The propagation of self similar convergent detonation wave in TATB-based explosive composition was studied both experimentally and numerically. The device constists in a $50 \mathrm{~mm}$ cylinder of TATB surrounded by an HMX tube. The detonation in HMX overdrives the detonation in TATB which adapts to the propagation velocity with a convergent front at centerline. We measured a curvature of $\kappa=-21.2 \mathrm{~m}^{-1}$ for propagation velocity of $8750 \mathrm{~m} / \mathrm{s}$, which extends the knowledge of the $\left(\mathrm{D}_{n}, \kappa\right)$ law. A wide ranged EOS/reaction rate model inspired from previous work of Wescott et al. was calibrated to reproduce both the run-to-detonation distance and the newly extended $\left(\mathrm{D}_{n}, \kappa\right)$ law for the 1D sligthly curved detonation theory. 2D Direct Numerical Simulations (DNS) were made on fine resolved mesh grid for the experimental configuration and for various driver velocities. The simulation reproduces the experimental data both qualitatively (overall detonation structure) and quantitatively $\left(\kappa=-25.4 \mathrm{~m}^{-1}\right)$.
\end{abstract}

\section{Introduction}

The insensitive high explosives (such as TATB-based compositions) are non ideal explosives: i.e. their detonation velocity is very sensitive to the local curvature of the front. This dependence can be modeled by the Detonation Shock Dynamics usually calibrated on slightly divergent steady detonations being propagated in rods. It has been demonstrated that on the center streamline the detonation obeys celerity-curvature law according to the quasi-steady 1D theory of slightly curved detonation. In order to extend both the celerity-curvature law to negative curvatures and the validity of DSD to convergent configurations, we present an experiment with a self-similar convergent detonation wave.

Experimental studies of convergent detonation, such as 1D spherical detonation or 2D merging fronts, are usually difficult to analyze because the propagation is not stationary which complicates the connection between local instantaneous velocity and curvature. In order to obtain the stationary constant wave with a negative curvature, we propose an experimental configuration (similar to that used by Souletis and Groux [1]) where the detonation in a cylindrical sample of TATB explosive is driven by an external tube of HMX. As the ideal detonation velocity (known as Chapman-Jouguet $\mathrm{D}_{C J}$ ) of HMX is much higher than that of TATB, the detonation is overdriven at constant speed in TATB and adopts a concave shape.

The goal of this study is to measure the concave shape profile of the established overdriven detonation wave and associated flow in order to extend the validity of the celerity - curvature law to slightly convergent detonation. A model is then calibrated to match this new point on the $\left(\mathrm{D}_{n}, \kappa\right)$ curve. Then, the comparison between the experiment and the direct numerical simulation will consolidate confidence in the newly calibrated model.

\footnotetext{
a e-mail: remy. sorin@cea.fr
}

This is an Open Access article distributed under the terms of the Creative Commons Attribution-Noncommercial License 3.0, which permits unrestricted use, distribution, and reproduction in any noncommercial medium, provided the original work is properly cited. 


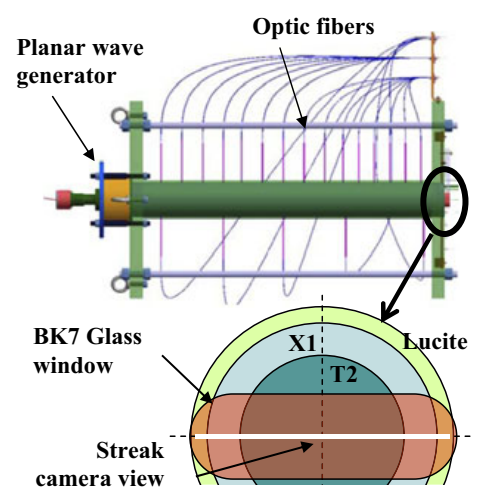

Fig. 1. Experimental device.

\section{Experimental device}

The experimental device consists of a cylinder of composition T2 (97\% TATB), of diameter $50 \mathrm{~mm}$ and length $600 \mathrm{~mm}$, surrounded by a $10 \mathrm{~mm}$ thick tube of composition X1 (96\% HMX), the whole contained in a $5 \mathrm{~mm}$ tube of lucite (cf. Fig. 1). The air gap at the interfaces is filled with EPOLAM to avoid jet formation. The detonation is ignited at an end using a plane wave generator. Souletis and Groux [1] showed that the structure of the detonation in the cylinder (the central Mach wave) is established once the detonation propagates over a length corresponding to 2.5 to 3 diameters. Thus, the length of the device is sufficient to ensure that the structure of the detonation is established when the front is recorded.

The chronometry of the detonation wave in the tube of HMX is recorded by electric and optical pins along two opposite meridian lines. It is corrected with various delay because of the equipment of measurements (reaction time of probes, transit time in wires...). Once the flow is established, at length $525 \mathrm{~mm}$, an X-radiographic image of the detonation front is recorded. The X pulse is produced with an $\mathrm{X}$-ray generator $(1.55 \mathrm{MeV}$ energy, focal beam of $1 \mathrm{~mm}$ and dose of $1 \mathrm{rad}$ within $1 \mathrm{~m}$ ) and the image is recorded on a series of couples of screens/films (consisting of OG2, LXFb or LXFf films, and Pb or BMS screens). In order to increase the Signal-to-Noise ratio, films of same kind are packed and the areal mass image is calculated from these stacking. An Abel inversion is then applied to obtain the tomographic reconstruction of the instantaneous density map of the flow (cf. Fig. 4). The shape of the detonation emerging on the back face is recorded by imaging a diameter with a mechanic-optics streak camera.

\section{Experimental results}

The shapes of the detonation emerging on the end-face of two experiments are shown on Fig. 2. The two profiles are quasi superimposed which proves that the phenomenon is very reproductive. The measured velocity in X1 in the tube $(8750 \mathrm{~m} / \mathrm{s})$ is quite close to the theoretical CJ velocity $(8780 \mathrm{~m} / \mathrm{s})$. The detonation shape of detonation is nearly flat except at the edges of the tube where, due to the side expansion, it becomes highly curved. This expansion leads to the oblique shocks in lucite and T2 of respective slope of $43.4 \pm 0.5^{\circ}$ (green line) and $44 \pm 0.5^{\circ}$ (blue line), very close to their theoretical values (respectively $42.3^{\circ}$ and $42.4^{\circ}$ ) calculated from oblique polar shock theory. One observes at $35 \mathrm{~mm}$ radius a stiffening of the profile recorded on Fig. 2. This stiffening corresponds to the interaction between the wave and the tube end whose corners are cut out at $45^{\circ}$ in order to ensure the assembly of the explosive inside the lucite.

Inside the $\mathrm{T} 2$ cylinder, the expansion of the detonation products of $\mathrm{X} 1$ creates an oblique non reactive shock with a sufficient pressure $(\sim 13 \mathrm{GPa}$, cf. Fig. 2$)$ to ignite the reaction in the TATB. A detonation is then induced and one observes a sudden change of the slope from $44^{\circ}$ to $62.3^{\circ}$ (orange 


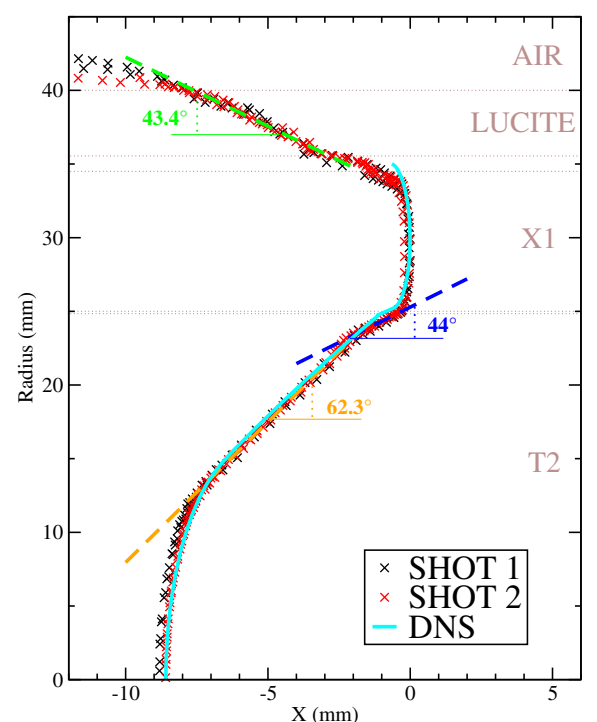

Fig. 2. Streak Camera Record and Numerical results (in cyan).

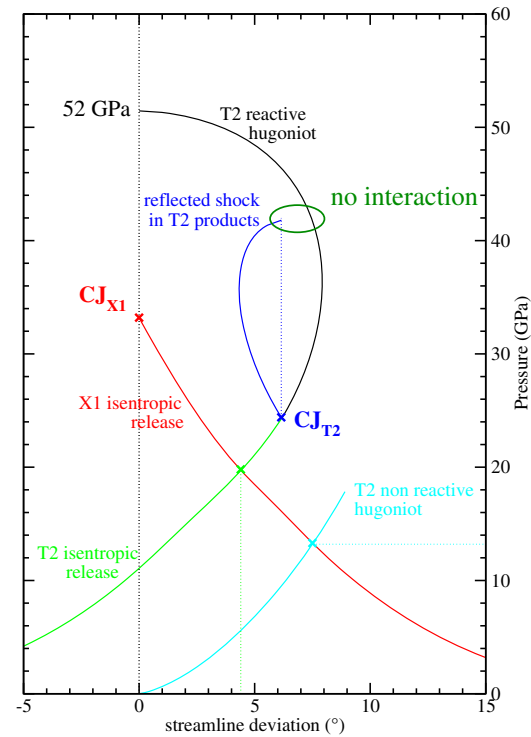

Fig. 3. Pressure deviation diagram.

line), located at radius $23.5 \mathrm{~mm}$. The estimated propagation velocity of the oblique detonation gives a value of $7754 \mathrm{~m} / \mathrm{s}$, which is close to the theoretical CJ value for this composition $(7751 \mathrm{~m} / \mathrm{s})$. The theoretical pressure-deviation diagram (cf. Fig. 2) proves that the detonation in $\mathrm{T} 2$ is not forced by the expansion of the detonation products of X1. Indeed, the pressure of the CJ point of T2 is always higher than the isentropic expansion of X1. Thus the oblique wave with slope equal to $62^{\circ}$ is an oblique detonation in $\mathrm{T} 2$.

Below radius $15 \mathrm{~mm}$, the detonation front changes and exhibits a concave form to adapt to the flow. On the central streamline, we measure a local curvature $\kappa=-21.2 \pm 5 \mathrm{~m}^{-1}$ and a normal velocity of $D_{n}=8750 \mathrm{~m} / \mathrm{s}$. From the streak camera record, a transmitted shock traveling at $7140 \pm 50 \mathrm{~m} / \mathrm{s}$ in the BK7 glass window was detected. From the BK7 hugoniot we deduced a pressure of $55 \mathrm{GPa}$, which corresponds to a shock reflected in detonation products of T2 starting from a pressure of $52 \mathrm{GPa}$ (the theoretical pressure of the curved detonation on the axis, cf. Fig. 2). Away from the centerline as the radius increases, the streamline deviation increases until the convergent detonation wave joins the oblique detonation (for a streamline deviation of $6^{\circ}$ ), at radius $13 \mathrm{~mm}$. On the pressure-deviation diagram, no solution exists between the overdriven convergent detonation (black) and the reflected shock from CJ point. Thus, no triple point should exist at the junction of these two detonations.

The tomographic reconstruction of the X-radiography is shown on Fig. 2 (upper side). This image gives access to the complete flow behind the detonation wave. As seen on Fig. 2, the shock wave in the lucite and the detonation in T2 are described. The precision of the image does not seem sufficient to see the inert shock in $\mathrm{T} 2$ at radius $25 \mathrm{~mm}$. Nevertheless, the principal structures of the flow, like the release waves, the direct and reflected shocks and the interfaces are visible on the image. The CJ expansion in $\mathrm{X} 1$ is seen on the image (grey triangle behind X1 detonation wave). This double expansion is enforced by the presence of EPOLAM at the two interfaces of the tube.

The convergent flow created in the T2 cylinder leads to a deviation of X1/T2 interface towards the axis. The deviation angle measured is approximately $4^{\circ}$, close to the theoretical value of $4.6^{\circ}$ on the pressure-deviation diagram. The X1/T2 interface abruptly changes its deviation angle from $4^{\circ}$ to $10^{\circ}$ when the reflected shock interacts with it, and thus creates a divergent flow. When approaching the axis, this reflected shock becomes difficult to detect. The streak camera records show a high pressure area at approximately $12 \mathrm{~mm}$ of the axis, which corresponds to the junction zone between main curved detonation and reflected shock. It is difficult to say whether the disappearance of the line comes from the blur related to the tomographic reconstruction or from the decreasing intensity of the shock. 


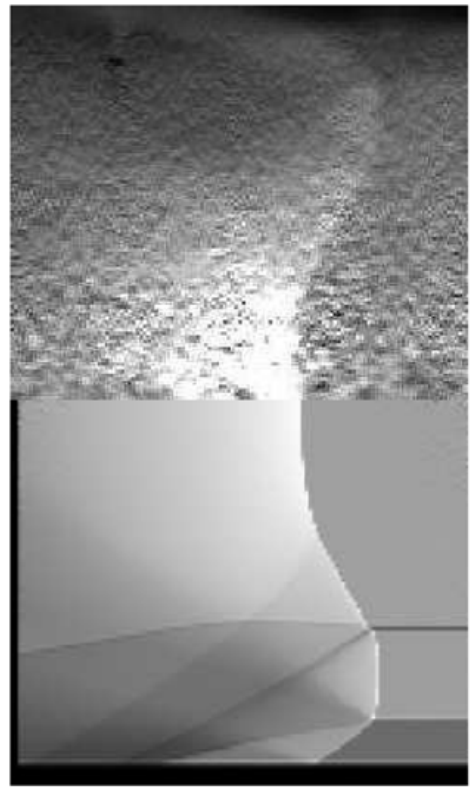

Fig. 4. Tomographic reconstruction of the X-radiography (upper side) and of the calculated pseudo-radiography (lower side).

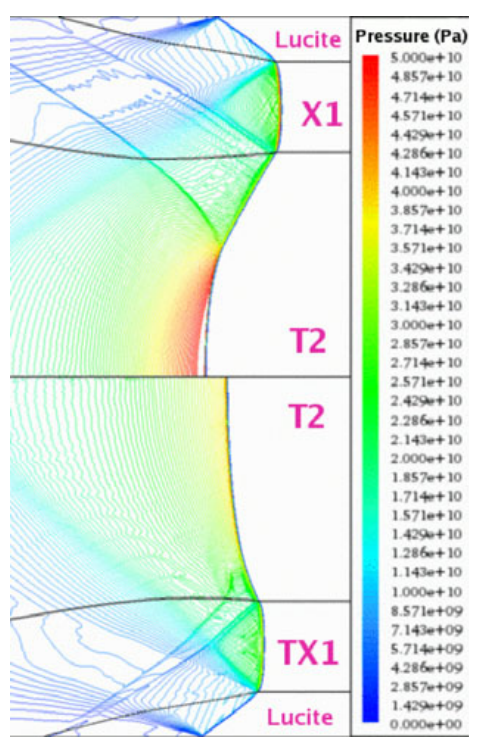

Fig. 5. Pressure field for X1 driver (upper side) and TX1 dirver (lower side).

\section{Numerical results}

We calibrated a model for the compositions X1 and T2 based on the wide range EOS/rate law model proposed by Wescott et al. [3]. The reactive equation of state is the ideal mixture of an unburnt phase (which reproduces the non-reactive hugoniot), with a burnt phase tabulated from the thermochemical code CARTE [2]. The rate law is then calibrated to reproduce both the run-to-detonation distance and the $\left(\mathrm{D}_{n}, \kappa\right)$ curve. We then use this model to perform $2 \mathrm{D}$ axi-symetric direct numerical simulation (DNS) on a regular $50 \mu \mathrm{m}$ mesh.

The simulated detonation profile is shown on Fig. 2 (cyan line) and the pressure field associated is shown on the Fig. 4 (upper side). Let us underline the extremely good correspondence between the measured and simulated profiles. The overdriven detonation in T2 is correctly represented, as well for the oblique detonation slope $\left(61.2^{\circ}\right)$ as for the curvature at the centerline $\left(\kappa=-25.4 \mathrm{~m}^{-1}\right)$. The main discrepancy with experiment is in the location of the reflected shock. The DNS over-estimates the radius ( $14 \mathrm{~mm}$ to the axis) where the three waves join.

A fine analysis of the reflected shock shows that the shock strength decreases when reaching the detonation fronts. Thus, the three waves are not connected through a triple point. At that point we suggest that the subsonic flow is stabilized via shocklets (cf. Guderley [4]) not described in simulation. Higher order numerical schemes would be necessary to describe this mode (cf. Skew and Ashworth [5]).

Figure 4 is the tomographic reconstruction of the experimental X-radiography (upper side) versus the tomographic reconstuction of the pseudo-radiography calculated from the density field obtained with the simulation (lower side). The structure of the flow observed on the radiography is entirely reproduced by simulation. In the DNS, the slopes of shock in lucite is equal to $36^{\circ}$. The calculated $\mathrm{X} 1 / \mathrm{T} 2$ interface shows deviations from $4^{\circ}$ towards the axis (after initial detonation) up to $9.6^{\circ}$ in the opposite direction (after the reflected shock).

Figure 6 represents the density (normalized with the initial density, i.e. the signal intensity just before the detonation front) versus distance along constant radius lines for the tomographic reconstruction of the radiography and the DNS. As seen on the Fig. 2, the location of the detonation fronts is correctly reproduces for all 4 radii extracted, despite the absence of the non-reactive shock 


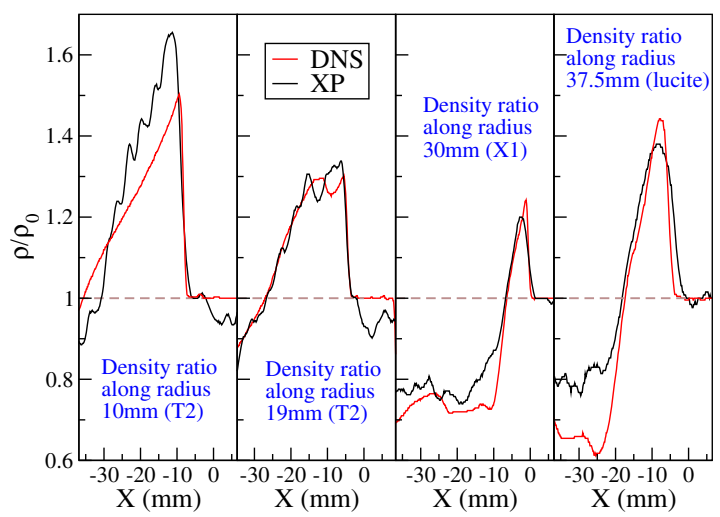

Fig. 6. Normalized density variation along different radii.

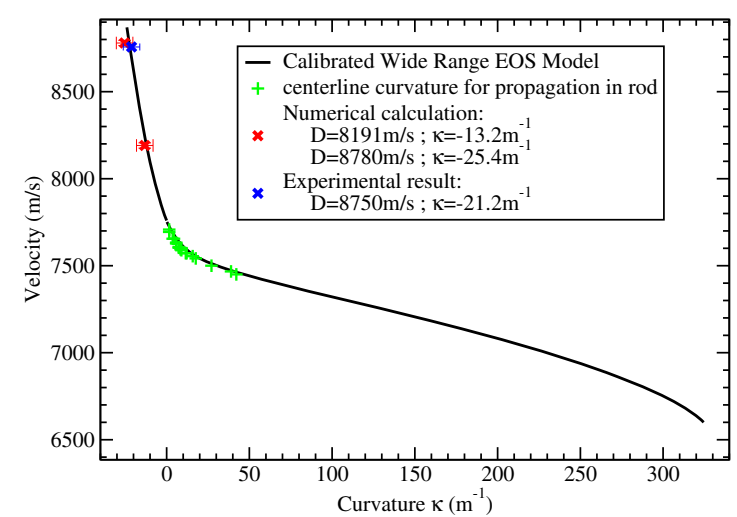

Fig. 7. $\left(\mathrm{D}_{n}, \kappa\right)$ curve for WR EOS/reaction rate model and central curvature in experiment (blue point) and calcultation (red points).

in the DNS. The two curves on the left side correspond to the density variation in Lucite and X1 (at radius 37.5 and $30 \mathrm{~mm}$ ). The two curves on the right side correspond to the density variation in the TATB-based explosive (at radius 19 and $10 \mathrm{~mm}$ ). We avoid the comparison on the centerline because the tomographic process disrupts the signal and the analysis becomes impossible. Moreover we had a gaussian blur to the pictures to smooth the extracted curves. The flow in T2 is correctly represented except that the model under-estimates the density just behind the detonation front. Moreover the reflected shock is nearer to the detonation front for the DNS, which confirms that the location of the three waves junction is over-estimated in the DNS. The WR EOS/rate law model can be modified to represent more closely the experiment, but in that case representative characteristics of the detonation, like the run to detonation distance, could be lost.

We have studied the detonation in T2 for other driver explosive, i.e. for different driver velocities. As expected, the higher the driver velocity, the lower the detonation curvature at centerline. Figure 4 represents the pressure field in the same geometric configuration but with two diffrent driver explosives (X1 for the upper side and TX1 for the lower side). TX1 is a mixed TATB-HMX-based explosive with CJ detonation velocity $(8290 \mathrm{~m} / \mathrm{s})$ located between those of X1 and T2. The main structures observed in $\mathrm{T} 2$ are the same (i.e., oblique detonation and weakly curved detonation joined with a reflected shock) except for the reflected shock location. We simulated different velocities for the driver (by varying the initial density of the HMX composition) and we saw that the reflected shock location is closer to the axis when the detonation velocity is increased.

On the Fig. 4, one can observe that the centerline curvature of T2 driven by TX1 $\left(\kappa=-13.2 \mathrm{~m}^{-1}\right)$ is higher than that of T2 driven by X1 $\left(\kappa=-25.4 \mathrm{~m}^{-1}\right)$. Figure 7 represents the $\mathrm{D}_{n}, \kappa$ curve used to calibrate the WR EOS/rate law model and the results obtained experimentally and numerically. As 
expected the DNS respects the theoretical curve. An experimental device producing convergent detonation waves was designed to validate DSD calculation with this prolonged $\left(\mathrm{D}_{n}, \kappa\right)$ law (cf. Matignon [6]). Comparison between DSD, DNS and experimental results confirms the reliability of the model to simulate low converging detonation front.

\section{Conclusion}

We designed an experimental device in order to obtain data to extend knowledge of $\left(\mathrm{D}_{n}, \kappa\right)$ curve to convergent detonation for TATB-based explosives. The detonation in a cylinder of TATB was overdriven by the established detonation of a tube of HMX. The stabilized detonation front was measured with the assistance of streak camera and X-ray radiographic picture. On the central steramline in TATB, the detonation adopts a convergent profile with a local curvature $\kappa=21.2 \pm 5 \mathrm{~m}^{-1}$ for a propagation velocity of $8750 \mathrm{~m} / \mathrm{s}$. The experiments were compared with DNS based on a Wide Range EOS model for the explosives. The DNS reproduces the whole flow structure behind the detonation both qualitatively and quantitatively.

\section{References}

1. Souletis and Groux, Continuous observation of mach bridge and mach phenomena, 8th International Detonation Symposium, Albuquerque, (1985).

2. Charlet, Turkel, Danel and Kazandjan, Evaluation of various theoretical equations of states used in calculation of detonation properties, Journal of Applied Physics 84, (1998).

3. Wescott, Stewart and Davis, Equations of state and reaction rate for condensed explosives, Journal of Applied Physics 98, (2005).

4. Guderley, Consideration on the structure of mixed subsonic-supersonic flow patterns, Technical Repport F-TR-2168-ND, Wright Field, (1947).

5. Skews and Ashworth, The physical nature of weak shock wave reflection, Journal of Fluid Mechanics 542, (2005) pp. 105-114.

6. Matignon, Sorin and Bozier, Detonation propagation of convergent front in IHE: comparison of DNS and DSD against experimental data, 14th International Detonation Symposium, Le Coeur d'Alène, (2010). 